\title{
Zeitstabilität der Nutzerwahrnehmung: Bewertung von Website-Inhalten
}

\author{
Dustin Witte ${ }^{1}$, Gerrit Hirschfeld ${ }^{2}$, Meinald T. Thielsch ${ }^{1}$ \\ Institut für Psychologie, Westfälische Wilhelms-Universität Münster ${ }^{1}$ \\ Fakultät Wirtschafts- und Sozialwissenschaften, Hochschule Osnabrück ${ }^{2}$
}

\section{Zusammenfassung}

In der vorliegenden Studie wurde untersucht, wie zeitlich stabil die Bewertung von Website-Inhalten ist. Insgesamt 212 Probanden (59\% Frauen) bewerteten jeweils eine von acht Websites dreimal in einem Zeitraum von 2 Wochen. Die Beurteilung des Inhaltes erfolgte mit dem Fragebogen zur Wahrnehmung von Website-Inhalten (WWI), die Websites wurden während der Befragung voll funktionsfähig präsentiert. Sowohl für die Gesamtscores als auch für die Subskalen Gefallen, Qualität \& Nutzen, Verständlichkeit sowie Glaubwürdigkeit fanden sich hohe Korrelationskoeffizienten zwischen den drei Messzeitpunkten. Die Wahrnehmung von Websites wird folglich stabil durch den WWI erfasst.

\section{Einleitung}

Der Inhalt ist das zentrale Merkmal von Websites und unterscheidet diese in besonderem $\mathrm{Maß}$ von anderen interaktiven Produkten. Dabei ist das Internet ein sehr schnelllebiges Medium, in dem Unternehmen und Institutionen einem enormen Wettbewerb ausgesetzt sind. Agarwal und Venkatesh (2002) benennen Inhalt als wichtigsten Website-Aspekt für bestimmte Geschäftsbereiche, während Palmer (2002) Inhalt als bedeutsamsten Faktor für den generellen Erfolg einer Website betont. Während die Bewertung von Websites im Bereich von Ersteindrücken - man benötigt z.B. nur $50 \mathrm{~ms}$, um zu entscheiden, ob eine Website als ansprechend empfunden wird (Tuch et al. 2012) - bereits gut erforscht ist, gibt es unseres Wissens bisher noch keine Studie, die die inhaltliche Bewertung von Websites über einen längeren Zeitraum untersucht hat. Diese Lücke soll durch die vorliegende Studie geschlossen werden. 


\section{Methoden}

\subsection{Probanden und Material}

Die Studie wurde online mit insgesamt 212 (davon 59\% weiblich) Probanden durchgeführt. Ihr Alter lag zwischen 16 und 70 Jahren $(M=46.82, S D=14.4)$, die durchschnittliche Interneterfahrung betrug 15 Jahre $(S D=4.5)$. Die Probanden wurden ausschließlich über das Online-Panel PsyWeb (https://psyweb.uni-muenster.de/) rekrutiert und per E-Mail zu den jeweiligen Messzeitpunkten eingeladen.

Als Material wurden acht möglichst unbekannte und zugleich prototypische Websites ausgewählt, die jeweils aus einer anderen Inhaltskategorie stammen (vgl. Thielsch 2008). Die Kategorien der Websites waren: E-Commerce, Suchmaschine, Corporate Website, ERecruiting, Download Site, E-Learning und Informationssite (zwei Websites aus dieser Kategorie, davon eine Mocksite). Es wurden die voll-funktionsfähigen Websites verlinkt.

\subsection{Fragebogen zur Wahrnehmung von Website-Inhalten}

Der Fragebogen zur Wahrnehmung von Website-Inhalten (WWI, vgl. Thielsch et. al. 2014) erfasst als einziger validierter deutschsprachiger Fragebogen die inhaltlichen Aspekte Gefallen, Verständlichkeit, Qualität \& Nutzen sowie Glaubwürdigkeit. Er besteht aus insgesamt 12 Items, je 3 pro Subskala (z.B. Gefallen: „Die Website weckt mein Interesse.“; Verständlichkeit: „Die Inhalte sind anschaulich aufbereitet.“; Qualität \& Nutzen: „Die Informationen sind qualitativ hochwertig.“; Glaubwürdigkeit: „Ich finde die auf der Website dargebotenen Informationen glaubwürdig."), die auf einer 7-Punkt-Likert-Skala mit den verbalen Ankern „stimme überhaupt nicht zu“ (1) und „stimme voll zu“ (7) zu bewerten sind. Cronbach's Alpha beträgt .88 für den Gesamtfragebogen und liegt für die Subskalen zwischen .71 und .90.

\subsection{Ablauf}

Jeder Proband wurde zufällig einer der acht Websites zugelost und bewertete nur diese zu allen drei Messzeitpunkten. Beim ersten Messzeitpunkt wurden zusätzlich zur Websitebewertung demographische Daten und Ausschlusskriterien (Farbfehlsichtigkeit, Darstellungsfehler der Websites) erhoben. Um die Benutzung der Website zu gewährleisten, bearbeiteten die Probanden eine Suchaufgabe. Die Itempräsentation erfolgte in randomisierter Reihenfolge oberhalb der voll funktionsfähigen Website.

Messzeitpunkt 2 wurde durchschnittlich 1,5 Tage nach Messzeitpunkt 1 beantwortet. Zwischen der Bearbeitung von Messzeitpunkt 2 und 3 lagen durchschnittlichen 12 Tage, entsprechend zwischen Messzeitpunkt 1 und 3 durchschnittlich 13,5 Tage. 


\section{Ergebnisse}

Die Ergebnisse zeigen, dass sowohl der Gesamtscore des WWI, als auch die Scores der Subskalen Gefallen, Verständlichkeit, Qualität \& Nutzen sowie Glaubwürdigkeit über alle drei Messzeitpunkte hinweg stark mit sich selbst korrelieren. Die Mittelwerte unterscheiden sich nicht signifikant zwischen den Messzeitpunkten (T1-T2: $p=.68$; T1-T3: $p=.55$; T1-T3: $p=$ $.84)$.

\begin{tabular}{|c|c|c|c|}
\hline & $r(\mathrm{~T} 1, \mathrm{~T} 2)$ & $r(\mathrm{~T} 2, \mathrm{~T} 3)$ & $r(\mathrm{~T} 1, \mathrm{~T} 3)$ \\
\hline WWI & $.89 * * *$ & $.88 * * *$ & $.84 * * *$ \\
\hline Gefallen & $.85 * * *$ & $.84 * * *$ & $.81 * * *$ \\
\hline Verständlichkeit & $.80 * * *$ & $.84 * * *$ & $.77 * * *$ \\
\hline Qualität und Nutzen & $.76 * * *$ & $.79 * * *$ & $.73 * * *$ \\
\hline Glaubwürdigkeit & $.74 * * *$ & $.70 * * *$ & $.68 * * *$ \\
\hline \multicolumn{4}{|c|}{ Anmerkungen. $N=212$ pro Messzeitpunkt; Abstand T1 zu T2: 1,5 Tage; } \\
\hline T2 zu T3; 12 Tage; T1 zu T3 & ge; $* p<.05$ & $p<.01 ; * * * p$ & $<.001$ \\
\hline
\end{tabular}

\section{Diskussion}

Die Ergebnisse der Studie sprechen dafür, dass der WWI den wahrgenommenen Inhalt einer Website sowohl auf Ebene des Gesamtfragebogens als auch auf Ebene der Subskalen stabil erfasst.

Die Stabilität der Wahrnehmung von Inhalten kann an dieser Stelle lediglich vermutet aber nicht belegt werden, da zur Bewertung des Inhalts nur ein Instrument verwendet wurde und eine Replikation der Ergebnisse mit anderen Instrumenten noch aussteht. Erst wenn die Ergebnisse dieser Studie auch bei multipler Operationalisierung erneut zu finden sind, lässt sich eine grundsätzliche Stabilität der Wahrnehmung von Inhalten annehmen. Unterschiede zwischen verschieden Website-Arten können aufgrund der Verwendung von nur einer Website pro Kategorie in dieser Studie nicht untersucht werden.

Hinsichtlich des WWI lässt sich aber festhalten, dass insbesondere Gesamtwert und die beiden Subskalen Gefallen und Verständlichkeit gute Retest-Korrelationen zeigen. Zieht man die Kürze der Skalen mit nur je drei Fragen in Betracht, so sind auch die Werte der übrigen beiden Skalen sehr akzeptabel. Der WWI (und seine Subskalen) hat sich damit unter Stabilitätsgesichtspunkten der Messung als effizientes und reliables Instrument zur Erfassung von Website-Inhalten bewährt. 


\section{Literaturverzeichnis}

Agarwal, R. \& Venkatesh,V. (2002). Assessing a firm's web presence: a heuristic evaluation procedure for the measurement of usability. Inf. Syst. Res., 13, 168-186. doi: 10.1287/isre.13.2.168.84

Palmer, J.W. (2002) Web site usability, design, and performance metrics. Inf. Syst. Res., 13, 151-167. doi: $10.1287 /$ isre.13.2.151.88

Thielsch, M. T. (2008). Ästhetik von Websites. Wahrnehmung von Ästhetik und deren Beziehung zu Inhalt, Usability und Persönlichkeitsmerkmalen. Münster: MV Wissenschaft.

Thielsch, M. T., Blotenberg, I., \& Jaron, R. (2014). User Evaluation of web sites: From First Impression to Recommendation. Interacting with Computers, 26, 89-102. doi: 10.1093/iwc/iwt033

Tuch, A. N., Presslaber, E. E., Stöcklin, M., Opwis, K., \& Bargas-Avila, J. A. (2012a). The role of visual complexity and prototypicality regarding the first impression of websites: working towards understanding aesthetic judgments. International Journal of Human-Computer Studies, 70, 794811. doi: $10.1016 /$ j.ijhcs.2012.06.003

\section{Kontaktinformationen}

Dustin Witte

dustinwitte@web.de
Gerrit Hirschfeld

Hirschfeld@wi.hs-osnabrueck.de
Meinald T. Thielsch

thielsch@uni-muenster.de

http://www.meinald.de/ 